\title{
Investigating Treatment Literacy on Antiretroviral Drugs (ARVs) and Nutrient Interaction Among People Living with HIV/AIDS (PLHIV)
}

\author{
Nthani D* and Hamoonga Mwiinga Bona \\ Department of Food Science and Nutrition, University of Zambia, Lusaka, Zambia \\ *Corresponding Author: Nthani D, Department of Food Science and Nutrition, \\ University of Zambia, Lusaka, Zambia.
}

Received: February 23, 2021

Published: March 20, 2021

(C) All rights are reserved by Nthani $D$ and

Hamoonga Mwiinga Bona.

\begin{abstract}
Antiretroviral drugs (ARVs) are potent life prolonging drugs; with potential interaction with food. The importance of taking ARVs consistently and appropriately can never be over-emphasized. For ARVs to be effective there must be regular food supply, a nutrition plan and continuous support system to ensure healthy eating [1]. Nutrition is therefore an essential component of ART interventions. However, the effects of food and ARV drug interaction has not been singularly researched in Zambia. The study objective was to determine the level and scope of information known (treatment literacy) amongst people living with HIV (PLHIV) about food-nutrient and ARV drug interaction. Specific objectives were to determine treatment literacy of PLHIV, determine whether PLHIV knew the meal and medication timing of their drugs and to determine PLHIV awareness of possible adverse effects of nutrition and drug interaction. The study was carried out in three (3) purposively chosen ART sites in Chongwe District, encompassing all HIV clients above twenty (20) years accessing health care and having been on ARV drugs for over 6 months. The study assumed a qualitative cross sectional descriptive study using open ended questionnaires with the aid of an audio tape recorder for recording responses. The collected data was transcribed and coded; themes were identified by way of content analysis for reporting. The results indicated that treatment literacy was inadequate both in quality and quantity of content. Additionally, drug-meal timing was not strictly followed. Further findings revealed step down and instructional format without reason given for actions. Overall, respondents were not aware of any drug-nutrient interactions, let alone expected side effects upon initiating medication. Respondents expressed desire for privacy and generally yearned for more information on HIV/AIDS. The findings also clearly showed lack of information on food and nutrition linkages to HIV/AIDS. In conclusion, nutrition being a cross cutting issue; its importance in the management of non-communicable and communicable diseases including HIV across different human physiological states and age groups cannot be over emphasized. Therefore, arising recommendations should be taken seriously into consideration not only by Chongwe District Health Management Team but also extrapolated nationwide in implementation.
\end{abstract}

Keywords: Treatment Literacy; Human Immunodeficiency Virus; Nutrition; Antiretroviral Therapy; Food-Drug Interaction

\section{Introduction}

Human immunodeficiency virus (HIV) is a virus which attack cells that help the body to fight infection, making a person more vulnerable $[2,3]$. The virus attacks the immune system and impairs the body's ability to fight infection. Overtime, HIV weakens a person's immune system by destroying important cells that fight disease and infection. If left untreated, HIV can lead to the acquired immunodeficiency syndrome (AIDS) disease. Some people who contract HIV do not show symptoms (asymptomatic phase) or become ill for long periods or even years. During this "asymptomatic 
phase" of the infection, the immune system becomes progressively weaker and other pathogenic microorganisms can take advantage of the "opportunity" presented by the weakened immune system to cause other illnesses such as pneumonia or tuberculosis (TB). The medical dictionary [4] defines AIDS as a severe disorder caused by the retrovirus HIV, resulting in defective cell- mediated immune response that is manifested by increased susceptibility to opportunistic infections and certain rare cancers especially Kaposi's sarcoma. Rang, Dale, Ritter and Flower [5] confirm that viruses are small infective agents consisting of nucleic acid (RNA or DNA) enclosed in a protein coat. They are not cells with no metabolic machinery of their own, obligate intracellular parasites and utilizing metabolic processes of host cells they infect to replicate. RNA retroviruses such as HIV contain an enzyme reverse transcriptase which makes a DNA copy of the viral RNA; which gets integrated into the host cell genome and directs the generation of new virus particles. The overall study objective was to determine the information base known about food-nutrients and ARV interaction amongst PLHIV. Specific objectives included determining treatment literacy of PLHIV, determining whether PLHIV knew the timing of their drugs in relation to medication and were able to gauge their awareness of possible nutrition and drug interactions. Twofold variables tested constituted independent education level and ART provider's training/capacity. Dependent variable constituted knowledge level of ART clients. The null hypothesis tested was that PLHIV were aware of the food and drug (ARV) interactions.

\section{Transmission and progression}

People get HIV through close contact with HIV-infected body fluids like blood, semen, or vaginal secretions, most commonly acquired during unprotected sex with a person who has HIV (sex without a condom or HIV medicine to prevent or treat HIV), or in infants born to an HIV-positive woman not taking antiretroviral therapy. Virtually all forms of sexual contact that exchange body fluids, except kissing, carry a high risk for spreading HIV. While the risk for contracting HIV through recipient anal or vaginal intercourse is higher than for oral sex, HIV can be passed to either person engaging in oral sex. Sharing HIV contaminated injection equipment (e.g., needles, syringes) is another common cause for new HIV infections. People who use injectable drugs should never share syringes. Abuse of alcohol and non-injectable drugs also in- creases the risk for HIV infection by lowering people's inhibitions and increasing the trading of sex for drugs or money.

There is considerable variability in disease progression. An initial acute influenza-like illness is associated with an increase in the number of virus particles in the blood, their widespread dissemination through the tissues, and seeding of lymphoid tissue with the virion particles. Within a few weeks, the viraemia is reduced by the action of cytotoxic lymphocytes. Acute initial illness is followed by a symptomatic free period during which there is a reduction in the viraemia accompanied by silent virus replication in the lymph nodes associated with damage to lymph node architecture and loss of CD4 lymphocytes and dendritic cells. The clinical latency (median of 10 years) comes to an end when the immune response finally fails and signs and symptoms of AIDS begin to appear; opportunistic infections which include neurological diseases, bone marrow depression and cancers [5]. In an untreated patient, death usually follows within two years.

\section{Nutrition and HIV}

Poor nutrition hastens progression from HIV status (being diagnosed positive) to acquired immune deficiency syndrome (AIDS) caused by a retrovirus known as immunodeficiency virus (HIV). The virus attacks the immune system and impairs the body's ability to fight infections [1]. Some people who contract HIV do not show symptoms or become ill for years, a condition referred to as "asymptomatic phase" during which period the immune system becomes progressively weaker and other microorganisms take advantage of the weakened immune system to cause illnesses such as pneumonia or tuberculosis (TB). Once opportunistic infections are evident, the client is said to have AIDS, the terminal end of HIV infection [2]. Progression rate of HIV infection to AIDS depends on the general health and nutrition status before and during the time of infection. Good health including desirable nutrition delays progression and improve the quality of life; providing reason for desirable nutrition care and support as part of comprehensive care and treatment of HIV [1]. Currently there is no effective cure for HIV but with proper medical care and nutrition management, it can be controlled. ARV drugs do not cure HIV, but merely suppress viral replication consequently reducing viral load in the body and mitigating undesirable effects. This keeps the immune system strong enough to fight off disease hence reducing morbidity and mortality 
to according to Piwoz and Preble [6]. People may take various medications to treat HIV, opportunistic ailments such as colds, malaria and intestinal parasites. More than forty (40) ARV drugs are approved worldwide to treat HIV. ARVs are divided into four categories namely: non-nucleoside reverse transcriptase inhibitors, nucleoside reverse transcriptase inhibitors, protease inhibitors and fusion inhibitors (entry inhibitors). They are usually given in combination (combination therapy) to produce a synergistic effect as a recommended method of treating HIV-positive clients. Most people who treat their HIV will take a combination of two or more of these drugs each day for the rest of their lives [7]. Some people with HIV also take herbal extract remedies and micronutrient supplements.

Adequate nutrition is important for maximizing benefits of antiretroviral drugs consequently increasing compliance with treatment regimens both of which are essential to prolong and improve quality of life for PLHIV, maintain the immune system, manage opportunistic infections as well as sustain healthy levels of physical activity. Evidence shows important links between HIV outcomes and nutrition. According to Piwoz and Preble [6], epidemiological studies show a strong relationship between nutrition and HIV efficacy, of which ARV drug use is greatly increased by sound nutrition. A review by AMPATH [8,9] using the academic model for prevention and treatment of HIV in Kenya found that patients enrolled on a nutrition supplement program while on Antiretroviral Therapy reported greater adherence to medication, fewer food related side effects and greater ability to satisfy increased appetites. The same study revealed that majority of patients experienced weight gain, recovered physical strength and were able to resume labour activities. Praphan and Phanuphak [10] stated that HIV infection can negatively impact food security and nutrition which in turn affects disease progression and treatment outcomes.

\section{ART and nutritional requirements}

Nutrition care is an essential component of antiretroviral therapy (ART) interventions, especially in resource limited settings. It helps improve the effectiveness of ART in supporting body defense system recovery and overall well-being. Poor nutrition care on the other hand reduces the body's ability to absorb medication, hence individuals may find it difficult to cope with the ART side effects [1]. Application of nutrition care management knowledge and skills of side-effects can help to minimize side effects and improve adherence to treatment. Heightened undesirable medication side-effects may affect food intake and nutrient absorption, a factor which can also reduce adherence to consistent and appropriate medications.

PLHIV take medication to suppress HIV replication alongside taking medications to treat opportunistic infections (OIs) caused by HIV, reduce manifestation of symptoms and treat other diseases. However, for ART to be effective, there must be regular food supply, a nutrition plan and continuous support system to ensure healthy eating. By reducing viral loads, antiretroviral therapy (ART) help to ultimately improve nutritional status. Drugs interact with certain nutrients, a factor which affects their efficacy and serve to encourage client's capacity for adherence to drugs arising from the positive effects felt. UNAIDS Policy Brief on HIV, Food Security and Nutrition [11] added that patients receiving Antiretroviral treatment, Nutrition Counselling and Support experienced decreased side effects, increased treatment adherence and overall health and nutritional status.

Medical treatment slows progress of HIV replication, emerging of opportunistic infection (OIs) and ease manifestation of symptoms [12]. On the other hand, undesirable interactions between drugs and nutrients also affect nutritional status negatively by reducing food intake through vomiting and diarrhea thus affecting nutrient utilization leading to weight loss and under-nutrition. Ultimately, interactions between drugs and food/nutrients can result in poorer health and nutritional status if not addressed properly and in time [13].

\section{ARVs, Their types and Mode of action}

ART drugs or medications treat HIV but do not cure or kill the virus; just reduce the viral load (amount of virus in blood stream) [14]. However, when taken in combination (referred to as highly active ART - HAART or Combination - cART); they prevent virus growth, a factor which slows down HIV disease as illustrated in HIV life cycle schematic diagram below. 


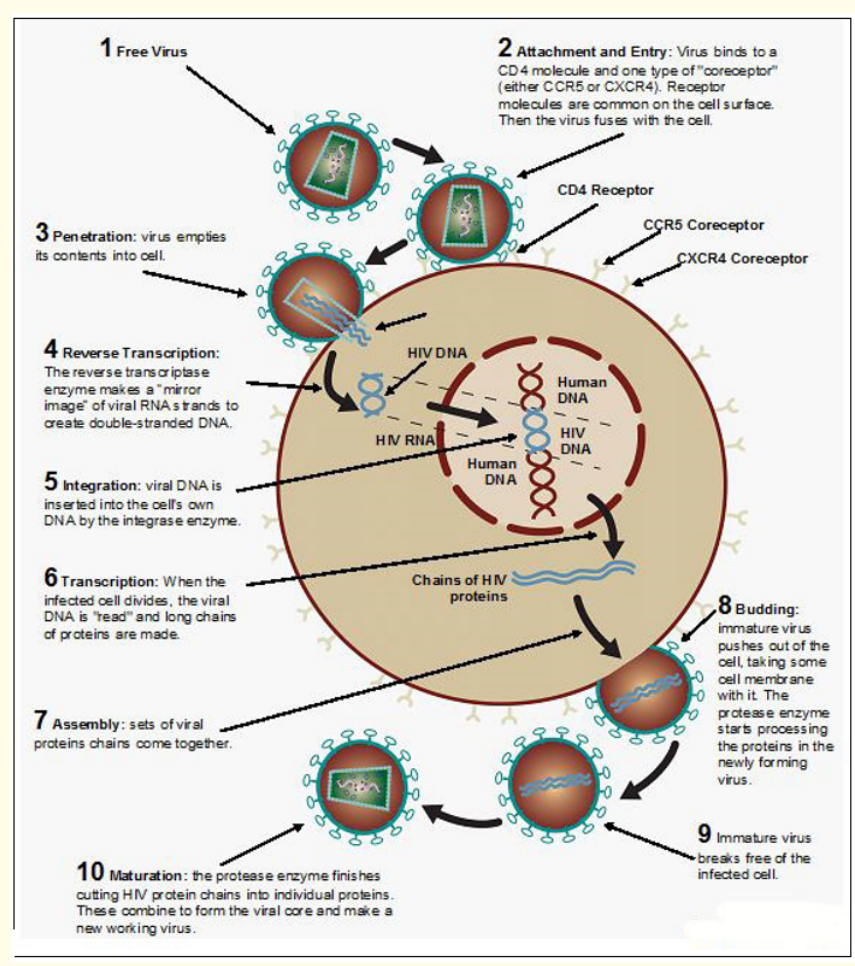

Figure 1

Source: HIV Drugs and the HIV Lifecycle (2019).

\section{Food-drug interaction}

Assertion by FANTA [15] states that medication can be affected by food. Therefore, understanding of drug and nutrition interaction plays a vital role in the provision of medical services available for patients. These must be assessed individually for the effect of food on medication action and the effect of medication on their nutritional status. These two aspects of medication use can be complicated by special diets, nutritional supplements, non-nutrients in food, alcohol intake, tube feeding etc. The national food and nutrition commission in the Nutrition Guidelines for the Care and Support of People Living with HIV and AIDS [1] states that drug-food interaction fall into categories, namely:

- Medications that may alter nutrient absorption, metabolism, distribution and excretion.

- $\quad$ Food may affect absorption, metabolism, distribution and excretion of medications.

- $\quad$ Some drugs may lower food intake or absorption
- Many medications may have diet restrictions (e.g. avoid milk and milk products when taken with tetracycline).

Medication side effects including loss of appetite/anorexia, taste changes, diarrhea, fatigue, depression, loss of sleep and pain; all factors likely to lower food intake.

It is incumbent upon health care providers to provide information on medication-food interaction to mitigate the side effects. (Appendix 1: Summary of interactions between food and ARVs commonly given in Zambia)

Overview of drug metabolism: Why nutrition matters

It is important to consider potential interactions between nutrition and xenobiotic substances foreign to the body including ARVs. Nutrition is the sum of all processes involved in the taking in of nutrients, assimilating these and using them for growth, repair and maintenance of the body [1]. The processes of nutrition include ingestion, digestion, absorption and metabolism (including transport to and from target tissues and functional utilization/activation of dependent systems). Although consideration of potential food-drug interactions is most often limited to questions of bioavailability (i.e., specific physical interference of drug absorption due to the presence or absence of food or specific food components in the gastrointestinal tract), metabolic fate of drugs, nutrients and other xenobiotics ultimately depends on nutritional factors. Raiten, Grinspoon and Arpadi [15] demonstrate the complexity of factors that affect aspects of nutrient bioavailability and other bioactive substances. In their article, they illustrate that drug-nutrient interactions including effects of drugs on nutritional status and the effects of nutritional status on drug absorption, efficacy or safety are more complex than just the physical relationship that occurs in the gastrointestinal tract. According to Wilkinson [16], pre-systemic xenobiotic metabolism occurring in the gastrointestinal tract and/or liver is an important predictor of drug delivery and efficacy. Wagner., et al. [17] asserts that a significant portion of drug metabolism occurs in the gastrointestinal tract via nutrient-dependent enzyme systems historically viewed as limited to the liver. The view of gastrointestinal tract as being only a potential barrier has evolved into one in which it is a pivotal locus of processes that affect bioavailability of xenobiotics to the general system. Xenobiotics metabolism consists of two essential phases: oxidation-reduction reactions involving mixed function oxidase (MFO) systems and 
the conjugation steps. Components of the gastrointestinal tract and liver tend to be more susceptible to nutritional effects. Yang, Brady and Hong [18] described several potential sites for dietary effects on the MFO systems. The authors explain that nutrients may 1) interfere with the genetic transcription and translation steps for synthesis of drug metabolizing enzymes; 2) influence the degradation of P450 mRNA and protein; or 3) directly influence MFO enzymes resulting in either increased or decreased enzymatic activity; demonstrating the delicate balance and exquisite responsiveness of the body to fluctuations in nutrient availability. This balance is further exemplified in a review by Ross and Zolfaghari [19] in which the importance of vitamin A on subsequent metabolism was described. The duo illustrates that pre-systemic metabolic systems that regulate availability of vitamin A to the body are subject to feedback regulation mediated by vitamin status (i.e., deficiency associated with changes in metabolism to increase availability and decrease elimination).

Specific examples of nutrient-drug interactions

For a given macro- or micronutrient, effect from an excess or deficiency may be paradoxical. As noted above, Ross and Zolfaghari [19] described the effect of vitamin A status on regulation of cytochrome P450 activity. Another example is riboflavin deficiency, which may result in either increase or decrease in the activity of particular components of the MFO system; effects which may be contingent on the insufficiency degree. Yang, Brady and Hong [18] affirm that mild riboflavin deficiency may result in decreased activity of NADPH reductase along with increased activity of several other enzymes; severe deficiency may result in decreased activity of several other MFO enzymes. Interestingly, riboflavin is a key component of the MFO systems. The relationship between riboflavin status and mitochondrial aberrations associated with ARV-induced lactic acidosis provides focus of interest. How a riboflavin deficiency induced by one drug may affect the safety and/or efficacy of other drugs such as ARVs has not yet been explored. To date none of these specific nutrient-drug relationships has been explored in the context of ARV use. Drugs can also affect specific elements of nutrient metabolism. For example, the therapeutic efficacy of several classes of drugs involves the ability to act as an anti-metabolite or antagonist for specific nutrients.
Non-nutrient components of food on drugs

Raiten [20] assert that food, particularly of plant origin, can provide essential nutrients and toxic substances. Factors other than essential nutrients (e.g., vitamins and minerals) have been identified in foods that influence the activity of drug-modifying conjugase and MFO systems. Brazier and Levine [21] and Izzo and Ernst [22] illuminate an additional concern in the growing use of dietary supplements, including herbal and botanical substances including the potential for adverse interactions with therapeutic drugs. Dresser, Schwarz, Wilkinson and Kim [23] elucidate the components of St. John's Wort, implicated in the induction of enzymatic components of MFO systems and subsequent effect on efficacy of certain classes of medications. Gorski, Huang and Pinto [24] provide echinacea as another example of a commonly used herbal supplement with potential to affect the safety and efficacy of therapeutic drugs. These examples emphasize the importance of understanding the effects of herbal/botanical treatments on the metabolism of therapeutic drugs when in use concomitantly. These issues become even more important in settings where the use of traditional medicines is the norm, with Zambia not being the exception. Caffeine consumption has been linked to decreased therapeutic response to psychoactive medications such as neuroleptics. This may result from an induction of liver metabolism or the formation of insoluble precipitates. Because caffeine consumption in the form of coffee, tea and soft drinks is common, these interactions may have clinical importance if such substances are consumed excessively. In the context of ARV drugs or other therapeutics that might be used by PLHIV, the range of caffeine intake beyond which problems might occur has not been defined. There is limited data regarding the effect of caffeine in HIV-positive patients. Caffeine has been found to exacerbate sleep disturbances in HIV-positive patients [25]. Tribble., et al. [26] report the fatal interaction among caffeine, ergotamine medication and protease inhibitors (PIs) while Baldwin and Ceraldi [27] provide that the more likely effect to be due to well-documented interaction between ergotamine and related compounds as used to treat severe headache and PIs than to an effect from caffeine per se.

\section{Food-drug interactions: Specific effects on ART}

Numerous studies show a direct interaction among food, herbal, botanical supplements and ARVs. Wanwimolruk and Prachayasitti- 
kul [28] documented specific food-ARV interaction involving garlic and the PI saquinavir. Long-term use (twice daily for 20 days) of garlic supplements was reported to significantly decrease plasma concentrations of saquinavir in otherwise healthy humans. Saquinavir was selected because it is a substrate of the P450 system. Whether these results can be generalized to other members of this class of drugs remains to be seen. Another commonly used traditional remedy particular in sub-Saharan Africa is Hypoxishemrocallidea, commonly known as the African potato. Mills., et al. [29] evaluated the impact of this botanical supplement together with Sutherlandia commonly used for relief of HIV-related symptoms on metabolism of ARVs. In these in vitro studies, both substances caused significant decreases in cytochrome P450 activity as well as reductions in the expression of $\mathrm{P}$ glycoprotein, an important component of drug transport. These authors concluded that these findings indicate the potential for increased drug toxicity, viral resistance, and treatment failure when these botanicals are used in conjunction with ART. As mentioned above, St. John's wort received considerable attention because of its possible effect on drug efficacy and safety through its induction of P450 system. The relevance of this concern to PLHIV was reinforced by Wanwimolruk and Prachayasittikul [28] who reported significantly reduced plasma concentrations of indinavir, another PI. Studies evaluated the relationship between and some selected nutrients (primarily macronutrients such as fat or protein) and the use of ARVs in terms of the pharmacokinetics and bioavailability of the drugs.

\section{HIV/AIDS treatment in Zambia}

Provision of antiretroviral therapy in Zambia began in late 2002 , although initially very few people could afford the monthly payments towards the drugs [30]. Free treatment provision started in June 2004 through involvement of many NGOs, churches and communities. At the end of 2011, 82 percent of the 510,000 people in Zambia needing ARV treatment were receiving it [31]; coverage estimation based on 2010 WHO guidelines. Although access to antiretroviral treatment was high and increasing among adults, coverage among children remained worryingly low, with only 1 in 3 children in need of treatment receiving it in 2011. However, the Ministry of Health further increased access to treatment by creating 68 new antiretroviral therapy (ART) sites in addition to supply- ing drugs to all existing ART sites nationwide in 2013. As a result of this, and support from the Global Fund, approximately 400,000 people had access to free treatment by late 2013 [32]. Unfortunately, update data for the purposes of reference was not available.

\section{Problem statement}

ARVs are potent life prolonging drugs with increased potential for interaction with food and nutrients, providing reason for taking them consistently and appropriately (including timing, taking them with or without food, and assortment of foods). The scope of food nutrients and ARVs interaction has not been singularly researched taking into account the available food base and habits in Zambia, but broadly bracketed in global folds. The National Food and Nutrition Strategic Plan for Zambia [11] states that the existence of insufficient information base on the nutritional status of PLHIV is a factor that constrains proper and solid guidance. Overall, there have not been extensive studies on the subject matter both in Zambia and elsewhere, a factor further constraining the basis upon which to anchor milestone counsel on client based knowledge on ARVs and food interaction. Even though the link between HIV/ AIDS and nutrition is well known and endorsed by the Zambian government, the content of nutritional education and counseling in HIV primary care is unclear. Furthermore, where provided, little is known about client adherence to health care provider advice about dietary behavior necessary for optimal management of HIV. It is assumed that patients on ART program know about basic sideeffects of ARVs and food interactions but the question still remains "do clients look for and spontaneously report changes experienced upon initiation of the regimen and documented?" It is not known at which stage of the treatment plan information is provided about side effects of ARVs, how this information is provided and how much clients understand the knowledge given. Additionally, there is lack of standardization in the practices to create awareness and educate HIV clients about side-effects of ARVs and food interactions. Altogether, this not only creates a public health problem but also compromises treatment outcomes affecting both pharmacodynamics (clinical or physiological effects) and pharmacokinetics (quantitative change in drug movement) of the drugs leading to more side effects. All undesirable side effects could be prevented with proper counseling, thus affecting adherence outcomes. This 
awakening provided impetus to investigate clients' knowledge levels of ARVs-food/nutrient interaction and their side effects. The research sought to investigate the knowledge levels of treatment literacy (understanding what HIV drugs are, why the drugs are needed and what they can and cannot do) supporting informed decision-making for personal health and community well-being of PLHIV, including respondents' awareness of possible drug and nutrition interactions. This is intended to inform and strengthen messaging for nutrition counselling and support of PLHIV.

\section{Justification}

As people with HIV live longer and new infection cases continue to be added each year, prevalence of the problem will continue escalating. Nutritional intervention is critical because of the unique combination of health consequences related to long-term ARV medication use, vicious cycle of malnutrition and immunity, and the threat of food insecurity among HIV-infected individuals. The study purpose (Knowledge, Attitude and Practice) was to determine knowledge levels of ART clients on drug and food interaction. The study was premised on the basis that the outcome would inform the need for understanding treatment literacy, client education and knowledge among PLHIV. Secondly the outcome would inform relevant line ministries and stakeholders for policy inclusion and provide information about perceptions of the side effects of ARVs-food/nutrient interactions used in planning Information Education Communication (IEC) approaches and also provide recommendations for improving the knowledge base and practices on food-nutrient/ARVs interactions and side effects amongst PLHIV. It will also help to bridge information gaps in HIV and nutrition policy, which may also be used to standardize food and nutrition protocols in nutrition counseling PLHIV on ART.

\section{Study design and population}

A qualitative descriptive cross sectional study design was employed owing to its usefulness for understanding human social experience, communication, thoughts, meaning, attitude, and processes, especially related to the interactions, relations, development, interpretation, movement and activity of people [33]. Exploration was based on Creswell [34] who explains that qualitative research is one where the inquirer makes knowledge claims based on multiple meanings of individual experiences and construct social meanings with an intent to develop a theory or pattern. Recognition was made of the fact that exploring sensitive issues is better articulated through interaction achieved through qualitative inquiry. Such an interaction offered an opportunity to capture individual's viewpoints, examine their everyday life constraints and securing rich descriptive data. In order to derive detailed information and useful insights, the depth and breadth of investigating of lived experiences phenomenon of patients taking ART; the complexities of sustained adherence required use of qualitative approach. The said study design was undertaken to determine knowledge levels on ARVs and food interactions on account of ascertaining understanding of holistic picture of factors surrounding antiretroviral drug treatment and food interactions among PLHIV. Given the sensitive nature of this study, use of in-depth open - ended interview question guides was deemed appropriate.

Sampling technique and sample size

The study was carried out in seven (7) ART sites out of which three (3) were static [Chongwe RHC, Kanakantapa RHC, Kasisi RHC] and four (4) served as outreach sites [Ngwerere Main RHC, Chalimbana RHC, Lwiimba RHC and Katoba RHC] in Chongwe District. More outreach sites were selected owing to the fact that the servicing of the outreach facilities was not consistent in terms of human resource. Chongwe District is a hub of political activities and external observers, situated $48 \mathrm{~km}$ east of Lusaka the capital city of Zambia. Overall, Chongwe District had an estimated population of 159 018, of which 81417 consisted of adult populations (15 years and above) [35].

The study population comprised HIV positive clients comprising men and women 20 years of age and above, were actively on ART drugs and accessing health care in respective health facilities. Lists of participants on active ART who were 20 years and above; selecting every third client meeting the inclusion criteria were compiled. This list provided a population sample from which a simple random sampling of names was drawn and a subset derived. The sample size achieved was twelve (12) respondents eligible for in-depth interviews. The interview mode of data collection allowed for col- 
lection of first-hand information from respondents seeking for attitudes, practices, feelings and thoughts expressed by self-other than by the second or third person forming the whole essence of a qualitative study. The inclusion criteria constituted clients who had confirmed HIV positive test result, been on antiretroviral treatment for over six (6) months and were willing to participate. Deliberate efforts were made to exclude patients who were too ill to participate, those who had not yet started taking antiretroviral treatment, were below 20 years or had just started taking antiretroviral treatment within the last six (6) months prior to the study.

Data collection and tools

In-depth interviews

Semi-structured in-depth interviews were administered to respondents in an informal and conversational way using a semi structured guide comprised of a list of key questions prepared in advance. To these questions responses enabled attaining of qualitative descriptions of respondents' world with respect to interpreting meaning. Flexibility to changes in sequence and forms of questions was allowed to accommodate follow up questions to answers given and stories as told by respondents. The conversations followed interpersonal natural situation based on themes under discussion in attempting to understand the world from "respondents' viewpoints" and unfold meaning of PLHIV experiences and uncover their lived world.

\section{Tape recorders}

Audiotape recorders were used to record the interviews which later got transcribed verbatim and translated from local languages into English. The transcripts were read independently to help develop a coding frame in readiness for analysis. Material about respondents' knowledge on food-ARVs interactions and perceived factors affecting treatment literacy were identified and used for systematic condensation.

\section{Data analysis}

In attempting to obtain sensible data whilst recognizing constraints of time limitation and resources, qualitative approach techniques were employed premised on Silverman [36] definition that states qualitative research as interpretation and inquiry into phenomenon with a purpose of understanding experiences from the respondents' points of view. A cross-section approach using rapid assessment procedure was employed to obtain data from respondents in selected health facilities and communities. This assumed holding in-depth interviews based on Wilson [37] who contends that qualitative research relies on firsthand knowledge under natural conditions and on unstructured data collection methods in which the investigator is the primary instrument or tool for data collection. The research utilized non-numerical data collected through interviews, observations and document analysis. After exploration of respondents" views, texts were given meaning and implication for recommendations were derived. Enquiry was systematic, planned and documented as expected.

The study concerned itself with issues of richness, texture and feeling of raw data developing insight and generalization out of the collected data. Individual interviews were conducted in a relaxed, non-formal way providing data rich in ideas, opinions and attitudes from respondents' view point while illuminating differences in perspectives between people. The process was based on Neuman [38] characterized by open procedure, determining "what existed" rather than "how much of it was there" allowing people to voice their opinions, perceptions and experiences the way they wanted to; aiming at understanding how issues were defined without imposing pre-formulated and rigid questions.

Exploratory inquiry process was employed to investigate applicability of drugs identification. Based on Ruane [39] who notes that exploratory research is typically conducted in the interest of "getting to know" or "understanding of a new phenomenon" used to gain an insight into the study concerns. Consequently, exploratory approach tended to utilize respondents' viewpoints that permitted attainment of "up close" first-hand information and in-depth understanding allowing "walking a mile" in respondents' shoes.

Content analysis of accrued data collected was used for the purpose of identifying and labeling or coding for analysis, analyzing both descriptive aspects (what the data was?) and interpretative (what the data meant). The process assumed a procedure for categorizing verbal or behavioral data for classification, summation 
and tabulation. It involved manual coding and classifying data (categorizing and indexing) so as to make sense of the collected data and highlighting important messages or findings. The narratives were analyzed separately to develop themes that were compared to the objectives to be coded. These were then translated into structural explanations for research findings development. Coding involved a process of combing the data for themes, ideas and categories and then marking similar passages of text with a code label so that they could easily be retrieved at a later stage for further comparison and analysis. This process made it easier to search the data making comparisons and identifying any patterns that required further investigation. The passages of text that were coded comprised sections of audio recording turned into written transcript. Any parts of the data that related to a code topic were coded with the appropriate label. All passages and chunks that were coded the same way and given the same label were judged to be about the same topic, theme, concept etc. The codes were given meaningful names that gave an indication of the idea or concept that underpinned the theme or category. If a theme was identified from the data that did not quite fit the codes already existing, then a new code was created. As the reading through the data set progressed, the number of codes evolved and grew as more topics or themes became apparent helping to identify issues contained in the data set. Most typically, when coding, some codes were already in mind and were also looking for other ideas that seemed to arise out of the data. When coding in this second, open minded manner, the process was premised on the provisions of the grounded theory suggesting asking questions about the data being coded: "What is going on? What are people doing? What is the person saying? What do these actions and statements take for granted? How do structure and context serve to support, maintain, impede or change these actions and statements?" [40].

\section{Validity and data reliability}

During data collection, there were factors that posed as threats to study validity and reliability. Measures were taken to secure validity and reliability. Validity in this context is another word employed for truth. It referred to the extent to which the data collection strategies and instruments measured what they purported to measure. Reliability on the other hand, referred to the extent to which studies could be replicated. This implied the degree of consistency with which instances could be assigned the same category by different observers or by the same observer on different occasions [41]. These two aspects were held in high esteem throughout the study.

Another factor that could threaten study validity and reliability was inadequacy of interviewer competency. To address this concern, pilot testing process was instituted to help improve questioning and probing techniques. Efforts were made to overcome the threat by practicing, listening to the tapes after the pilot testing and also after each interview to help overcome possible biases. During the interviews, efforts were made to listen carefully to the respondents' answers and elaborations. Respondents were given enough time to elaborate their answers with remarks such as, "Do you have anything else you want to tell me? In addition, probes were used to obtain more information. Consistency of information was checked by paraphrasing what the respondents said, for instance, "If I get you well, you said.....

Interview guides were pilot-tested on patients accessing health facilities but not participating in the study to avoid fatigue and bias in responses. Pilot testing of the guide was essential to check on clarity of questions, questioning techniques during the interviews and establishment of favorable rapport.

This stage took care of finding the right balance between openended and focused interviewing, asking leading questions, repeating questions, failure to listen closely and probe when necessary, failure to judge answers and asking vague or insensitive questions. Follow up discussions on experiences accrued were carried out with some of the informants who indicated whether the interview guides were serving the intended purpose. As such it was discovered that more information was needed hence the additional questions were added. Time was planned for this step and note taken of revisions resulting from this stage in ensuing checklists.

\section{Ethical Considerations}

Ethical clearance was sought from the University of Zambia Biomedical Ethics Committee and Chongwe District Health Of- 
fice. Written consent was sought from individual respondents by show of signature before participating in the study. Respondents were debriefed on the aims, nature and purpose, data collection procedure as well as their role in the study. Assurance was made that their personal data would be kept confidential by way of using codes and not names. Information was kept confidential by assigning identification file names to respondent interviews in the transcripts and not linking responses to any particular name. Respondents were assured that as soon as the report was approved, all draft scripts and notes would be disposed by way of burning under strict supervision. Further, as soon as all the interviews were fully transcribed to satisfaction, the records were destroyed. Monetary compensation to respondents was not possible because of a strict budget. Respondents were informed during debriefing that they would not be paid allowances as the interviews were conducted within normal operational hours for the respective health facilities.

\section{Findings and Discussion}

Treatment literacy

The Southern Tier AIDS group [42] states that treatment literacy entails an education program designed to help people living with HIV or AIDS to understand, manage and navigate the treatment options available to enable informed decisions generation. STAMP asserts that one-on-one and group counseling sessions should be held between PLHIV and ART providers to discuss HIV treatment and possible side effect management options. It is important that individuals living with HIV are helped to talk with their ART providers about various aspects of their experiences. Most importantly, treatment literacy programs should contain information on medication, HIV and the immune system; CD4 and viral load, when to start treatment, side effects, adherence, having a baby etc. These aspects provided the basis upon which questions on knowledge and treatment literacy and responses guided the discussions.

\section{Knowledge on Medication}

All respondents without exception described their medication by color and shape and not by names as evidenced by the typical quote below:
“... yes, I know it is medicine for HV. I used to drink this type 3 times a day, but now I only drink it once at 20:00hrs every night............ (Laughs, then continues in a small voice). I don't know the name (.... laughs again) but I know how it looks like", (then touching a pinkish cloth) says "it looks like this, like here......" (Pointing to a specific colour in the clothe)

[55 years old female respondent, 5 years on medication]

And another respondent said: "I only know that it is RV (misnomer). The ones I was taking in the beginning looked brownish like this (pointing at brownish khaki colour), but I was changed to the ones which look whitish like".

[40 years old female, 7 months on drugs]

For respondents who tried to remember something of the drug name either said something completely different or came out all wrong as evidenced in the quote below

"I am taking nevirapine and these white ones. I take 2 tablets at 20:00hrs and 1 in the morning. I take one white and one blue".

[47 years old male farmer, 1 year and 2 months on drugs]

Information on the above respondent's card showed that the client was on Emtricitabine (FTC), Tenofovir (TDF), Efavirenz (EFC) (Atripla) and not nevirapine as stated. This caption illustrates the fact that the client would not be able to demand for the right drug should s/he run out of medication and happen to be in another location where there was no client card for reference, an often possible occurrence. This typical scenario presents complications in the management of HIV in cases where every ART providing center is mandated to provide drugs to clients in emergency situations. In the event that the client does not know the actual correct drug name required, $\mathrm{s} / \mathrm{he}$ stands high chances to be given the wrong drug if not well described. Suffice to note that drugs from different manufacturers keep changing in both the shape and color implying that these attributes are not always reliable. Being on a wrong drug would have far reaching negative implications, not only metabolic complications but also most importantly attendant side-effects and 
drug resistance. Thus knowing the drug name is very important in the management of HIV/AIDS; not only relying on size, color and shape descriptions. The issue of not knowing the drug name is further complicated by inability to read as evidenced by the quote below.

"(laughs nervously), you know us who ended school in grade 7 reading is difficult. It's called nerseatm or never what, I don't know...... I take one once a day and septrin too".

[43 years' male farmer, 6 years on medication]

This caption emphasizes the importance of taking every aspect of treatment literacy seriously by ART providers and communicating it correctly to clients accordingly. Knowing the drug by descriptions of size, colour and shape is not a reliable proxy as various manufacturers use different characteristics and combinations differently accordingly.

\section{Knowledge of side effects}

Respondents' knowledge of the side effects was largely inadequate. Evidence showed that their knowledge base on side effects was either premised on beliefs or what they had heard from friends or the community as illustrated below.

"When I started treatment I used to have bad dreams and feel dizzy. I was told that the drugs I had started were causing the problems by my friends and so I had to stop taking the medicines (default) for a while...........

\section{[43 years' male farmer, 6 years on medication]}

Inadequate knowledge base entailed that respondents didn't know what to expect from taking the prescribed drugs, neither did they know what to do when side effects manifested; providing fertile ground for patient education. Lack of information from reputable sources places clients in very precarious positions to the extent that patients listen to uninformed informants. This represents danger for some respondents like the one above in stopping taking the drugs as required (defaulting), a factor which leads to other undesirable consequences (adherence issues). With so much misinformation circulating from various sources, it is important that health workers take time to explain what the client should expect as the medication is initiated so that they do not fall prey to misinformation. Respondents also expressed concern about taking medicines on empty stomach. They reported that they experienced discomfort including feeling dizziness, diarrhea, taste changes, loss of appetite, nausea and sometimes vomiting in varying degrees and assortment combinations. They unanimously expressed the fact that these experiences affected their food intake and the accompanying nutrient absorption in one way or another a factor that would compromise nutritional status. Consequently, in the event that they experienced the undesirable taste changes, advice to add any flavor enhancers such as salt, sugar, spices, vinegar or lemon to stimulate the taste buds, increase the taste acuity and musky any unpleasant flavors were never given. For these reasons they were somewhat hesitant to commerce taking the prescribed ARV drugs especially in the wake of poverty and hunger.

"I usually do not have food to eat at home so I am rather scared to take the drug. I have often experienced the feeling of vomiting when I take the RV on an empty stomach. I also cannot afford to keep feeling dizzy amid such a busy schedule for the day".

[43 years' male farmer, 6 years on medication]

\section{Knowledge on When to start Treatment}

The study revealed that respondents were not taken through any form of information schedule, a factor which placed them further at the mercy of misinformation. None of the respondents remotely knew about the criteria used for initiating ART. The response below indicate that the respondent did not have the right information concerning when they were supposed to start treatment.

Question: "When are you supposed to start taking ART?"

Response: "When and/or if you are found to be positive"

[47 years old male farmer, 1 year and 2 months on drugs] 
The Ministry of Health protocols [2] states that ART is supposed to be initiated using set criterion in both adults and adolescents as reflected below.

Clinical staging III and IV, CD4 count $<350$ cells/mm3, HIV positive partner in discordant couple, HIV positive with Hepatitis B virus infection and those with active tuberculosis as well as every pregnant woman

[Adult and Adolescent Antiretroviral Therapy protocol 2010 page 22].

As a matter of human right, HIV positive individuals are supposed to know when to start the treatment; being fully equipped with fair understanding about why some people even when found to be positive are not immediately started on drugs. This information base should help in positive living or self-care practices such as for HIV clients to know the reasons for either being put on ART straight away or for being deferred especially in instances where they come from the same home. This would avoid sharing of drugs and combat dropout's rates from the therapy.

\section{Knowledge on Viral Load and CD4 count}

The AIDS info Clinical Guideline Portal on plasma HIV-1 RNA (viral load) and CD4 count monitoring [43]. UNAIDS [44,45] state that "HIV RNA (viral load) and CD4 T lymphocyte (CD4) cell count are the two surrogate markers of antiretroviral treatment (ART) responses and HIV disease progression used to manage and monitor HIV infection. The most important use of viral load is as a marker of response to ART to monitor the effectiveness of therapy after initiation of ART.

The CD4 cell count provides information on the overall immune function of an HIV-infected patient. The measurement is critical in establishing thresholds for the initiation and discontinuation of opportunistic infection prophylaxis and in assessing the urgency to initiate ART". None of the respondents knew the difference between viral load and CD4 cell count. Some clients expressed themselves thus.
"Am not sure though I think CD4 count is the number of HIV viruses (tudoyo) in your body but I don't know what viral load is".

The level of treatment literacy was generally not satisfactory, even if the respondents knew some aspects of the treatment literacy package. This status quo clearly showed that treatment literacy was far below par of what they were supposed to know especially considering how long some of them had been on medication. Knowledge on CD4 and viral load would help ART clients understand the progress made and appreciate more the benefits of ART. This would give them a baseline against which they can measure progress made and give themselves health targets to achieve against their CD4 count and viral load readings. Furthermore, this would give them a better understanding of the disease prognosis.

Knowledge on drug and meal timing

Literature states that knowledge on drug and meal timing is important in providing information about the nutrition implications of drugs and effective management of the ensuing implications. Andrew McLachlan and Iqbal Ramzan [46] wrote about meals and medicines stating that "food and its constituents may have a significant effect on both the rate and extent of drugs absorption after oral administration". Understanding the effect of meals on medicines enables health professionals to advise patients about the appropriateness of ingesting medicines with respect to the times and composition of meals. The drugs can interact with food and nutrients affecting the drug efficacy and nutritional status. For example, a meal containing high fat enhances bioavailability of tenofovir. Knowledge on drug-nutrient interactions which take into consideration the drug/meal timing aims at improving medication efficacy, preventing nutritional status deterioration and ensuring adherence to drugs.

Responses to the question about how clients took their drugs in relation to drug-food timing and why they took them that way revealed that ART clients were largely not counselled by ART providers on the importance of correct drug-meal timing. Holding counseling session with clients would facilitate a process where opportunity would be accorded to enable ART clients understand 
why they had to follow the drug-meal timing strictly. Better adherence to drug uptake would facilitate improved drug efficacy, reduced manifestation of side effects and ultimately improved treatment outcomes. It is important to place clients in a position where they understand that each drug was different and taken differently from the other; thereby improving treatment literacy. Some of the responses attained were that every medication was supposed to be taken with food; clearly a factor which was not always true because food could either increase drug availability in circulation or reduce its effectiveness.

Drug meal timing

All respondents were apparently on Atripla drug at the time of the study. It was reported that appropriate time for taking drugs was chosen by respondents themselves based on recommendations from their ART providers. The respondents reported that they took drugs according to the usual family meal timing and the drug time revolved around that meal timing which would either be just a little while after or before the meal or even within the recommended 1-2 hours as reflected in the quote below.

"I take after eating, I only consider the drug time. If I eat early then I have to wait for 20:00hrs and if I cook late, I take the meds before food but I really don't like eating late because we sleep early".

[40-year-old female participant, 7 months on drugs]

Atripla $^{\mathrm{TM}}$ is a Food and Drug Commission (FDC) tablet containing three antiretroviral medications consisting of efavirenz, emtricitabine, in combination with tenofovir [47]. Since there is increased exposure to efavirenz and tenofovir when taken with a high fat meal, recommendation is that Atripla should be taken on an empty stomach or 2 hours after a main meal or an hour after a low fat light snack. The following response represents a typical scenario.

"......but I know that on taking any drugs, you need to have eaten. Uh uh! All drugs need food".

[47-year-old male, 1 year 2 months on drugs]
From the above responses, it was evident that the recommended drug and meal timing was not followed. Evidently, all respondents took the medication at the agreed time but without regard to the meal timing. It's very important that the drug meal timings are followed to improve on the drug's effectiveness and reducing the impact of side-effects such as toxicity as when the drug is taken with a high fat meal. The provision of timely and appropriate advice about the possible effects of meals on medicines and the importance (or lack) of the timing of meals and medicines is an important issue impacting on the quality use of medicines and the patients' quality of life.

\section{Reason for timing}

Side effects arising from Atripla include dizziness, trouble with sleeping, drowsiness, and trouble with concentrating; all reduced by taking the tablet on an empty stomach at bedtime. All respondents had their own reasons for drug-meal timings; based on own experiences, understanding or convenience. Some reported to being advised at the health facility. None of the respondents reported taking the drugs according to the recommended drug-meal timing. Ideally patients were supposed to understand why their medication was timed as such to improve on the practice of adherence and management of the side effects. Below is a reflection of some responses to the question on reason for taking the drug at the specified time in relation to meal timing.

"I was never told about how to take my medication......... (umm). You do not need to be told on that one, (uhh) you just need to know that those drugs are too strong".

[47-year-old male, 1 year 2 months on drugs]

Another reflection was as follows:

"There is no specific reason given for taking the medicine at the stated time, I was just told to choose the time that I would be taking my drugs by the ART provider. So I chose 12:00 hrs and they said no, choose the most appropriate time".

[43 years' male farmer, 6 years on medication] 
In this scenario, the respondent was not told anything about how to take the medication; rather assumed that all medication was supposedly taken with a meal.

On probe about timing, the following is an exception of the responses.

No, they never told me whether I should take at night or during the day. They never even gave me the reason, so I just chose night time because I would be going to sleep and would feel the negative effects less when I am asleep".

[43 years' male farmer, 6 years on medication]

In this scenario the respondent was encouraged to choose an appropriate time for when to take the medication but without being given a reason why. It's not only the drug-meal timing that is important to follow but also an understanding of why the drug (in this case Atripla) was to be taken at night for enhanced adherence and lessening the manifestation and impact of the side effects. For instance, drowsiness and dizziness could cause serious problems for clients driving and working with heavy machinery therefore, if taken during the day has the potential to incapacitate productive work.

\section{Awareness on drug nutrient interaction}

Information provided on Atripla packages caution consumption against alcohol, grape fruit and its juice and high fat foods intake. It is said that the drug also interacts with ginkgo biloba and St. John's wort [48]. All the respondents, without exception reported lack of knowledge with regard to drug-food interaction. In response to the question on specific side-effects knowledge with regard to drugnutrient interaction, the following were some of the responses.

"At first when I took the medicines before eating, I used to feel dizzy and nauseated [explains with eyes closed and distorts the face]. That is when I reasoned that it's better to eat first. I don't know if there is any connection".

[47-year-old male, 1 year 2 months on drugs].
Awareness of drug-nutrient interaction is very important as the information helps the patient to make informed decisions about side effects and how to remedy them where possible, besides helping in adherence for positive treatment outcomes.

On being asked about any foods that they may have been stopped or avoid since starting drugs. This was intended to check whether the foods they have been cautioned on corresponded with the foods contraindicated for the drugs indicated on the package elicited the following response.

“... they stopped me from taking alcohol and smoking because it was said that these two do not work well with the medication".

\section{[43 years' male farmer, 6 years on medication]}

Apparently all the respondents were cautioned against alcohol and smoking which do correspond with the medication instructions, but only 2 respondents indicated that they were cautioned against high fat foods attributed to their condition being compounded with hypertension and diabetes. Alcohol and smoking had been implicated in many non-communicable diseases and given the already compromised immune system in HIV/AIDS, would place them at higher risk of pulmonary incidences, organ system failures and cancers. High fat meals contra-indicate in all patients on Atripla but in this case only respondents with known hypertensive and diabetic conditions were cautioned. As mentioned above tenofovir and efavirenz in Atripla increases in its effectiveness with high fat meal/diet.

Information gaps and needs

The need for privacy and correct information about the disease and treatment options and outcomes cannot be over-emphasized. The PLHIV are supposed to have confidence in the system and feel free to discuss matters affecting their health individually. Respondents expressed need for more information, detailed screening by trained personnel and a separate dispensary and screening away from the general population because of the desire to ask questions about their medication in privacy. A separate dispensary would also reduce waiting times in long queues. Below are some of the expressed views. 
"We need to be taught more on HIV/AIDS and the benefits of ART adherence. It's not enough, they should be teaching us more about HIV/AIDS when we come to get our medicine......... They should provide a place for us to be taught more about HIV".

[55 years' female, 5 years on drugs]

Additional probing about the hesitation to seek attention revealed the following:

"We always find long queues justifying the spending of more time and that is discouraging".

[55 years' female, 5 years on drugs]

With regard to information types given by ART providers, the following is the except response:

“They don't tell us what to expect. We get information from friends. Like the time I defaulted, they were very annoyed but it was their fault because they didn't tell me anything before".

[43-year-old male farmer, 6 years on drugs]

Additional discussion revealed disappointment in the client disposition as reflected below:

They blame us for the disease...... It is not enough to just keep giving us medicines. It is not enough at all (shaking his head and looking dejected) ...... They should have specialized clinicians and nurses for us HIV patients.

Consequently, further discussions revealed that

"We are not comfortable to ask questions about our concerns because staff seem to have a lot of work. At the window you can't even ask any questions, it's too open and there are lots of people around". Group education is also not the best option because you cannot ask personal concerns for fear of being stigmatized.

[43-year-old male farmer, 6 years on drugs]

The outcome of the above quote clearly shows that opportunities to allow clients to ask personal concerns was not happening.
They cannot ask the care provider at the dispensary about issues concerning their medication since it's an open public place. As a result, they were not given enough information about issues affecting their health. Just being seen in the group automatically raises insinuations that one is HIV positive.

\section{Conclusion}

The study revealed multiplicities of factors that need to be taken into consideration when providing ART for comprehensive and effective outcomes. All the respondents confirmed that they were not provided with any information with regard to ARVs including their benefits neither were they given possible options from which to choose. Adequate clear and comprehensive information on the disease, treatment options and possible side effects need to be given to the patients by ART providers. However, implementation of this requirement possess a number of challenges including inability to provide personalized information across different age groups, language, time availability, literacy levels against the shortage of human resource and privacy. The clients expressed that all they ever expected was to be given medicines just like they get drugs for any other illnesses as part of health care service provision. However, suffice to note that ARVs are lifelong and possess great potential for cumulative effects especially in the face of defaults. Additionally, information given by the ART providers should be educational rather than instructional. The findings indicate that treatment literacy was grossly inadequate in both the quality and quantity of content. The drug and nutrient interactions as well as drug-meal timing was not followed. Further the findings revealed that the kind of information given followed a step down format; instructional in nature without reason being given for the recommended action. Respondents were instructed to take Atripla at night but were not given reasons why night time was preferable. Providing education on the drug name should not be brushed aside as unimportant especially for the illiterate populace. Evidently, in the absence of correct information dissemination, aspects of sharing ignorance across different divides of the population become eminent. There is urgent need for powers that be to work out modalities to try and address the identified shortfalls in the design and direction of interventions to promote treatment literacy among PLHIV for effective health outcomes. 
Generally, respondents were not aware of drug-nutrient interactions, let alone what to expect as side effects when they started medication. Further the respondents expressed desire for privacy and more information on HIV/AID. The findings also clearly showed the lack of knowledge about food and nutrition information and linkages to HIV/AIDS. Nutrition being a cross cutting issue; its importance in the management of communicable and non-communicable diseases across the different physiological states and age groups cannot be overemphasized [49]. Calls for full-fledged nutrition counselling employing instructional format were discouraged and deemed not very useful. Not explaining the full implications of defaulting ARVs for any length of time is very cardinal for positive health benefits to be accrued for the rest of one's life in the face of taking medication correctly. District HIV/AIDS and nutrition program officers should work in synergy to ensure that HIV and nutrition management protocols were readily available and followed as intended. The HIV/AIDS and nutrition program officers should appraise the quality and adequacy of HIV and nutrition care quarterly in all the ART sites and extrapolated nationwide in implementation because experiences are likely similar across the nation. The null hypothesis tested proved that PLHIV were not aware of the food and drug (ARV) interactions.

Appendix

\begin{tabular}{|c|c|c|c|}
\hline Medication & Nutrition guidance & Avoid & Possible side effects \\
\hline \multicolumn{4}{|c|}{ Antiretroviral drugs (ARVs) } \\
\hline Abacavir (ABC) & $\begin{array}{l}\text { Take with or without food, } \\
\text { but taking with food reduces } \\
\text { side effects. Alcohol increas- } \\
\text { es levels of side effects }\end{array}$ & Alcohol & $\begin{array}{l}\text { Nausea, vomiting, fever, allergic reaction, anorexia, abdominal pain, diar- } \\
\text { rhea, anaemia, rash, hypotension, pancreatitis, dyspnea, weakness and } \\
\text { insomnia, cough and headache }\end{array}$ \\
\hline $\begin{array}{l}\text { Emtricitabine } \\
\text { (FTC) }\end{array}$ & $\begin{array}{c}\text { Take before bedtime, with or } \\
\text { without food }\end{array}$ & Fatty food & $\begin{array}{l}\text { Dizziness, drowsiness, insomnia, abnormal dreams, impaired concentra- } \\
\text { tion, headache, diarrhea, nausea, rash, skin discoloration }\end{array}$ \\
\hline $\begin{array}{l}\text { Lamivudine } \\
\text { (3TC) }\end{array}$ & Take with or without food & Alcohol & $\begin{array}{l}\text { Nausea, vomiting, headache, dizziness, diarrhea, anaemia abdominal pain, } \\
\text { nasal symptoms, cough, fatigue, pancreatitis }\end{array}$ \\
\hline Stavudine $(\mathrm{d} 4 \mathrm{~T})$ & Take with or without food & Alcohol & $\begin{array}{c}\text { Nausea, vomiting, diarrhea, peripheral neuropathy, chills and fevers, appe- } \\
\text { tite loss, rash, rash, stomatitis, anaemia, headaches, pancreatitis, lipodys- } \\
\text { trophy, hyperlipidemia, increased liver enxymes }\end{array}$ \\
\hline \multicolumn{4}{|c|}{ Non-nucleoside reverse transcriptor inhibitors (NNRTIs) } \\
\hline Efavirenz (EFV) & $\begin{array}{l}\text { Take with or without food } \\
\text { just before bedtime }\end{array}$ & $\begin{array}{l}\text { Alcohol, high } \\
\text { fat meals }\end{array}$ & $\begin{array}{l}\text { Elevated blood cholesterol levels, elevated triglycerides, rash, dizziness, } \\
\text { anorexia, nausea, vomiting, diarrhea, dyspepsia, abdominal pain, flatu- } \\
\text { lence }\end{array}$ \\
\hline Etravirine (ETV) & Always take after a meal & $\begin{array}{l}\text { Taking on } \\
\text { an empty } \\
\text { stomach }\end{array}$ & $\begin{array}{c}\text { Sever skin rash, allergic reactions, immune reconstitution inflammatory } \\
\text { syndrome (IRIS), gain or loss of body fat, tingling, numbness or pain in } \\
\text { hands or feet (peripheral neuropathy) }\end{array}$ \\
\hline $\begin{array}{l}\text { Nevirapine } \\
\text { (NVP) }\end{array}$ & Take with or without food & $\begin{array}{l}\text { St. John's } \\
\text { wort }\end{array}$ & $\begin{array}{c}\text { Nausea, vomiting, rash, fever, headache, skin reactions, fatigue, stomatitis, } \\
\text { abdominal pain, drowsiness, paraesthesia, high hepatoxicity }\end{array}$ \\
\hline \multicolumn{4}{|c|}{ Protease inhibitors (PIs) } \\
\hline $\begin{array}{l}\text { Atazanavir/ } \\
\text { Ritonavir } \\
(\mathrm{ATV} / \mathrm{r})\end{array}$ & Take with food & $\begin{array}{l}\text { Alcohol, St. } \\
\text { John's wort }\end{array}$ & $\begin{array}{c}\text { Diarrhoea, gas, nausea, fat maldistribution, vomiting, stomach pain, kidney } \\
\text { stones, hyperglycemia, lactic acidosis (rare), hyperlipidaemia }\end{array}$ \\
\hline
\end{tabular}




\begin{tabular}{|c|c|c|c|}
\hline $\begin{array}{l}\text { Lopinavir/ } \\
\text { Ritonavir } \\
\text { (LPV/-r) }\end{array}$ & Take with or without food & $\begin{array}{l}\text { St. John's } \\
\text { wort }\end{array}$ & $\begin{array}{l}\text { Nausea, vomiting, weakness, diarrhea, headache, dizziness, abdominal } \\
\text { pain, fever, fever, diabetes, anorexia, hepatitis, jaundice }\end{array}$ \\
\hline Ritonavir (RTV) & Take with food & $\begin{array}{l}\text { St. John's } \\
\text { wort }\end{array}$ & $\begin{array}{c}\text { Nausea, vomiting, diarrhea, taste changes, hepatitis, jaundice, weakness, } \\
\text { appetite loss, abdominal pain, fever, diabetes, pacreatitis, headache, dizzi- } \\
\text { ness, possible increased risk of lipodystrophy, increased triglyceride levels, } \\
\text { increased uric acid, increased liver enzymes }\end{array}$ \\
\hline \multicolumn{4}{|c|}{ Integrase strand transfer inhibitor (INSTI) } \\
\hline $\begin{array}{l}\text { Dolutegravir } \\
\text { (DTG) }\end{array}$ & Take with or without food & & $\begin{array}{l}\text { Allergic reaction, liver problems, gain or loss of body fat, immune reconsti- } \\
\text { tution inflammatory syndrome (IRIS, trouble sleeping, fatigue, headache }\end{array}$ \\
\hline \multicolumn{4}{|c|}{ Antibacterial Medications for TB } \\
\hline Rifampicin & $\begin{array}{c}\text { Take on an empty stomach, } 1 \\
\text { hour before or } 2 \text { hours after } \\
\text { a meal. Supplement with } 10 \\
\text { mg vitamin } B_{6} \text { daily }\end{array}$ & Alcohol & $\begin{array}{c}\text { Gastrointestinal irritation, anaemia, jaundice, pancreatitis, altered taste, } \\
\text { anorexia }\end{array}$ \\
\hline Rifabutin & $\begin{array}{l}\text { Take with or without food } \\
\text { (with food if stomach is } \\
\text { upset) }\end{array}$ & High-fat food & $\begin{array}{l}\text { Urine discoloration, neutropenia, leukopenia, thrombocytopenia, rash, } \\
\text { diarrhea, headache }\end{array}$ \\
\hline
\end{tabular}

Appendix 1: Summary of interactions between food and ARVs commonly given in Zambia.

Source: Republic od Zambia Ministry of Health Directorate of Clinical Care and Diagnostic Services. 2016. Zambia Consolidated Guidelines for Treatment and Treatment of HIV infection. Lusaka, Zambia: MOH.

http://www.hivpolicywatch.org/duremaps/data/guidelines/ZambiaARTguidelines 2013.pdf; WHO: 2016.

\section{Bibliography}

1. Zambia Ministry of Healthy, National food and nutrition commission and Foods and Nutrition Assistance III project (FANTA). "Nutrition guidelines for care and support of people living with HIV". Lusaka: Ministry of health (2017).

2. Ministry of Health. "Adult and Adolescent Antiretroviral Therapy protocols" (2010)

3. HIV.gov: Overview about HIV/AIDS: What are HIV and AIDS (2020).

4. The American Heritage Medical ${ }^{\mathrm{M}}$ Dictionary (2007).

5. Rang HP., et al. "Rang and Dales Pharmacology". $6^{\text {th }}$ Edition. Edinburgh: Churchill Livingstone (2007).

6. Piwoz E G and Preble E A. "Links between HIV outcomes and Nutrition”. HIV/AIDS guide for Nutrition Support (2001).

7. Medically reviewed by Zara Risoldi Cochrane, Pharm.D., M.S., FASCP - Written by Stephanie Watson - Updated on April 24, 2020 Antiretroviral HIV Drugs: Side Effects and Adherence.
8. Elizabeth Byron., et al. "AMPATH-Integrating nutrition security with treatment of people living with HIV: Lessons from Kenya" (2006).

9. Joe Mamlin. "Academic Model for the prevention and treatment of HIV/AIDS" (2006).

10. Praphan and Phanuphak. "Policy Brief: HIV, Food Security and Nutrition-WFP, WHO and UNAIDS" (2006).

11. National food and nutrition strategic plan for Zambia. 20112015 (2011).

12. NFNC. "Nutrition guidelines for care and support of people living with HIV/AIDS". Zambia (2011).

13. FANTA. "Food and Nutrition Technical Assistance, Nutrition and HIV/AIDS: A training Manual for Nurses and midwives" (2008).

14. International Association of AIDS Care Providers (IAPAC). "What is Antiretroviral Therapy (ART)?" Fact Sheet 403 (2014). 
15. Daniel J Raiten., et al. "Nutritional considerations in the use of ART in resource limited settings-Consultation on nutrition and HIV/AIDS in Africa: Evidence, Lessons and recommendations for Action" (2015).

16. Wilkinson G R. "The effects of diet, aging and disease states on pre-system elimination and oral drug bioavailability in humans". Advanced Drug Delivery Reviews (1997): S13-S31.

17. Wagher D., et al. "Intestinal drug efflux: formulation and food effects". Advanced Drug Delivery Reviews (2001): S13-S31.

18. Yang CS., et al. "Dietary effects on cytochrome P450 xenobiotic metabolism and toxicity". FASEB Journal 6 (1992): 737-744.

19. Ross AC and Zolfaghari R. "Regulation of hepatic renal metabolism: Perspectives from studies on vitamin A status". Journal of Nutrition 134 (2004): 269S-275S.

20. Raiten. Nutrition, pharmacology and toxicology: a dialectic. In Massaro E. Ed. Handbook of toxicology. Boco Ration. FI. CRC Press (1997).

21. Brazier N C and Lewis M A. "Drug-herb interaction among commonly used conventional medicines: A compendium for health care professionals". American Journal of Therapeutics 10 (2003): 163-169.

22. Izzo A A and Ernst E. "Interactions between herbal medicines and prescribed drugs: A systematic review". Drugs 61 (2001): 2163-2175.

23. Dresser G K., et al. "Coordinate induction of both cytochrome P450 3A and MDRI by St. John's Wort in Healthy subjects". Clinical Pharmacology and Therapeutics 73 (2003): 41-50.

24. Gorski J C., et al. "The effect of Echinacea (Echinacea, purpurearoot) on cytochrome P450 activity in vivo". Clinical Pharmacology and Therapeutic 75 (2004): 89-100.

25. Food-Medication Interactions by Zaneta M. Pronsky M.S. 18th Edition (2015).
26. Tribble Gregg., et al. "Fatal egotism induced by an HIV protease inhibitor". Headache 42 (2002): 694-695.

27. Baldwin Z K and Ceraldi C C. "Ergotism Associated with HIV Antiretroviral protease inhibitor therapy". Journal of Vascular Surgery 37 (2003): 676-678.

28. Wanwimolruk Sampon and Prachayasittikul Virapong. "Cytochrome P450 enzyme mediated herbal drug interactions". EXCLi Journal 13 (2014): 347-391.

29. Mills E., et al. "Impact of African herbal medicines on antiretroviral metabolism". AIDS 19 (2005): 95-97.

30. Lewis S. "Zambia: A Constellation of change and commitment in response to AIDS" (2003).

31. UNAIDS. Global Report: AIDSinfo (2012).

32. United Nations. MDGs and Beyond: MDG 6 Factsheet (2013).

33. Dreber H M. "The effect of caffeine reduction on sleep quality and wellbeing in persons with HIV". Journal of Psychosomatic Research 54 (2003): 191-198.

34. Creswell JW. "Research Design: Qualitative and quantitative approaches". Thousand Oaks: Sage (1994).

35. Zambia Statistics Agency, Ministry of Health (MOH) Zambia, and ICF. Zambia Demographic and Health Survey 2018. Lusaka, Zambia, and Rockville, Maryland, USA: Zambia Statistics Agency, Ministry of Health, and ICF (2019).

36. Silverman D. "Interpreting qualitative data". $3^{\text {rd }}$ Edition. London: Sage Publications (2006).

37. Wilson H S. "Introducing research in Nursing: Cumming" (1993).

38. Neuman LW. "Social Research Methods: Qualitative and Quantitative approaches". $4^{\text {th }}$ Edition. London: University of Winscosin at Whitewater (2000). 
39. Ruane J M. "Essentials of research methods: A guide to social science research". USA: Blackwell Publishing (2005).

40. Charmaz K. "Grounded theory methods in social justice research" (2003).

41. Malterud K. "Qualitative research: standards, challenges, and guidelines" (2001).

42. STAMP. "Treatment literacy in HIV/AIDS” (2015).

43. “AIDS Info Clinic guideline portal on plasma" (2014).

44. WHO. "Scaling up Antiretroviral therapy in resource-limited settings: Guidelines for public health approach" (2002).

45. Madhu Vaipayee. "UNAIDS: Current practices in laboratory monitoring of HIV infection". Indian Journal of Medical Research 134.6 (2011): 801-822.

46. Andrew Mclachlan and Iqbal Ramzan. Meals and Medicines. Faculty of Pharmacy. University of Sydney. NSW (2006).

47. Atripla ${ }^{\mathrm{TM}}$ Package insert. "Bristol Myers Squibb and Gilead Sciences". Foster City, Califonia US.

48. East, Central, and Southern African Health Community (ECSAHC), Food and Nutrition Technical Assistance Project (FANTA), and LINKAGES Project. "Nutrition and HIV/AIDS: A Training Manual for Nurses and Midwives. Arusha, Tanzania: ECSA-HC" (2008).

49. HIV/AIDS: A guide for Nutritional care and support. $2^{\text {nd }}$ Edition. Food and Nutrition Technical Assistance Project, Academy for Education Development, Washington DC (2004).

\section{Assets from publication with us}

- Prompt Acknowledgement after receiving the article

- Thorough Double blinded peer review

- Rapid Publication

- Issue of Publication Certificate

- High visibility of your Published work

Website: www.actascientific.com/

Submit Article: www.actascientific.com/submission.php

Email us: editor@actascientific.com

Contact us: +919182824667 